\title{
Características Estereológicas del Hígado de Rata (Rattus norvergicus) sometidas a Menopausia Inducida por Ovariectomía
}

\author{
Stereologic Characteristics of the Liver of Rats (Rattus norvergicus) \\ Submitted to Ovariectomy-induced Menopause \\ "Erick Trujillo; ${ }^{* *}$ Bélgica Vásquez \& ${ }^{* * * *}$ Mariano del Sol
}

TRUJILLO, E.; VÁSQUEZ, B. \& DEL SOL, M. Características estereológicas del hígado de rata (Rattus norvegicus) sometidas a menopausia inducida por ovariectomía. Int. J. Morphol., 29(4):1470-1478, 2011.

RESUMEN: Durante la menopausia ocurren cambios hormonales, como disminución de estradiol y aumento de las hormonas folículo estimulante y luteinizante. En mujeres premenopáusicas la fibrosis y esteatosis hepática es menor que en mujeres postmenopáusicas, mejorando cuando se administra terapia hormonal de reemplazo. El objetivo del estudio fue determinar aspectos cuantitativos del hígado de ratas sometidas a un modelo experimental de menopausia inducida por ovariectomía. Utilizamos 10 ratas Sprague-Dawley (Rattus norvergicus) adultas, sanas. Las ratas fueron pesadas y divididas en dos grupos (ovariectomía bilateral=Grupo Ovx y control=Grupo Sham). A los 60 días fueron sacrificadas y retirado el hígado, obteniéndose un trozo de cada hígado. Se realizaron 5 cortes de $4 \mu \mathrm{m}$ y se tiñeron con HE. Se analizaron 5 campos por lámina. Se usó el test M42. Los parámetros estereológicos considerados fueron: Nv, Vv, Sv y número total (Nt) de hepatocitos y sinusoides hepáticos. El peso promedio del grupo Ovx y Sham fue 300,8 g y 285,8 g respectivamente. La Nv de hepatocitos de ratas sometidas a cirugías Sham (CS) y Ovx (CO) fue de $6,89 \times 10^{5} / \mathrm{mm}^{3}$ y $7,70 \times 10^{5} / \mathrm{mm}^{3}$, respectivamente. La Vv de hepatocitos CS y CO fue de $71,92 \%$ y $80,48 \%$, respectivamente. La Sv de hepatocitos CS y CO fue de $260,61 \mathrm{~mm}^{2} / \mathrm{mm}^{3}$ y $273,64 \mathrm{~mm}^{2} / \mathrm{mm}^{3}$, respectivamente. El volumen promedio de hígados CS fue de $8,40 \mathrm{~mm}^{3}$ y el Nt de hepatocitos promedio fue 5,79 x $10^{6}$. El volumen promedio de los hígados CO fue $9,28 \mathrm{~mm}^{3}$ y el Nt de 7,15 x $10^{6}$. Respecto a los sinusoides, la Nv en el hígado CS y CO fue de $2,81 \times 10^{5} / \mathrm{mm}^{3}$ y $2,64 \times 10^{5} / \mathrm{mm}^{3}$, respectivamente. La Vv de sinusoides en CS y CO fue de $16,74 \%$ y 16,46\%, respectivamente. La Sv de sinusoides en ratas CS y CO fue de $49,40 \mathrm{~mm}^{2} / \mathrm{mm}^{3} \mathrm{y}_{45,21} \mathrm{~mm}^{2} / \mathrm{mm}^{3}$, respectivamente. El Nt de sinusoides CS y CO fue en promedio 2,37 x $10^{6}$ y 2,45 x $10^{6}$, respectivamente. La ausencia de estrógeno puede generar fibrosis y esteatosis hepática como lo señalan otros autores, pero también, cambios en la organización y proporción de los componentes del hígado, factores a considerar durante el diagnóstico y pronóstico de alteraciones hepáticas.

PALABRAS CLAVE: Menopausia inducida; Ovariectomía; Hígado; Estereología; Rattus norvergicus.

\section{INTRODUCCIÓN}

La menopausia, es el cese permanente de la menstruación después de la pérdida de la función ovárica (Santoro, 2002). Los cambios hormonales importantes que se producen, corresponden a una disminución de estradiol circulante con aumento concomitante de las hormonas folículo estimulante y luteinizante (Lip et al., 1997).

Estudios experimentales sugieren que el estrógeno es un poderoso antioxidante que participa en el metabolismo de los ácidos grasos. Por lo tanto, cuando sus niveles se encuentran disminuidos, también sus efectos protectores. Así, Lip et al. reportaron que tres a cinco años post menopausia natural, produce un aumento significativo de los triglicéridos, colesterol total y LDL colesterol y dentro de las primeras seis semanas después de la ovariectomía. Respecto a la función hepática, Di Martino et al. (2004), Codes et al. (2007a) de- mostraron que las mujeres premenopáusicas presentaban una menor cantidad de fibrosis hepática que las mujeres postmenopáusicas y cuando se administraba terapia hormonal de reemplazo, el hígado mostraba parámetros similares al grupo premenopáusico.

Nemoto et al., (2000) demostraron que la esteatosis hepática se hizo evidente de manera espontánea en ratas y en ratones con deficiencia de la enzima aromatasa, por tanto, carecen de la capacidad intrínseca de producir estrógeno. Yasuda et al. (1999) demostraron que al aplicar terapia de estradiol, existe una reducción de la fibrosis y de la esteatosis hepática. Concluyen, que la progresión de la lesión hepática, independientemente del sexo, puede deberse, al menos en parte, a la disminución de la producción de estrógeno y/o la falta de respuesta a las acciones de este.

\footnotetext{
" Programa de Magíster en Morfología Universidad de La Frontera. Universidad de Talca, Chile.

** Universidad Autónoma de Chile.

**** Universidad de La Frontera, Chile.
} 
Existen evidencias que demuestran que la disminución de la función ovárica se asocia con aumentos en los niveles séricos de citoquinas proinflamatorias como las interleucina 1 y la interleucina 6 y también el factor de necrosis tumoral alfa y que las concentraciones normales de estrógenos inhiben el aumento o la secreción espontánea de tales citoquinas (Shimizu, 2003; Codes et al., 2007b). Camargo et al. (1997), señalaron que estas citoquinas promueven la regeneración hepática mediante la inducción de la proliferación de hepatocitos.

Según Shimizu (2007), las hormonas sexuales pueden desempeñar un papel importante en la progresión de enfermedades hepáticas crónicas como la esteatosis o la fibrosis. Las evidencias epidemiológica y clínica indican fuertemente que los estrógenos, en particular, el 17 b-estradiol tiene un papel preponderante en la distribución general y en el metabolismo del tejido adiposo, la deficiencia de éstos favorece el acúmulo de tejido adiposo en los tejidos, especialmente en el hígado, por la disminución de la actividad lipolítica de éste (Mayes \& Watson, 2004; Lavoie et al., 2011).

Según Angulo (2002), los estudios clínicos, bioquímicos y por imágenes son de valor en la evaluación diagnóstica de los pacientes con hígado graso no alcohólico y otros trastornos del hígado. Sin embargo, la biopsia hepática sigue siendo el medio más sensible y específico de proporcionar información de diagnóstico y pronóstico. El esclarecimiento reciente de los mecanismos que conducen a la progresión de la enfermedad hepática sugiere una variedad de nuevos objetivos metodológicos que permitan una mejor aproximación para conocer las causas y consecuencias de estas alteraciones.

El hígado es estructural y funcionalmente un tejido complejo, a pesar de que el hepatocito constituye en un tejido normal casi el $80 \%$ del tejido hepático, existen al menos otra docena de células que desempeñan importantes funciones (Malarkey et al., 2005).

Weibel et al. (1969) informaron un 83,1\% de hepatocitos en el hígado de ratas Wistar y Valenca et al. (2008), una densidad de volumen de $48 \%$ y densidad de número de 42,2 $\pm 0,98 \times 103 \mathrm{~mm}^{3}$. Blouin et al. (1977) informaron un 77,8\%, de hepatocitos en hígado de ratas Sprague-Dawley. Por otra parte, Águila et al. (2003) en estudios experimentales en ratas, alimentadas con una dieta alta en lípidos, determinaron que el número de hepatocitos se encontraba entre 56 y $62 \%$.

Respecto a los sinusoides hepáticos, estudios morfométricos han sido realizados en diversas especies. Blouin et al. reportaron en ratas Sprague-Dawley un 10,6\% y Ragonha et al. (2006) determinaron que en el hígado de ratones los sinusoides corresponderían a un 18,3\%.
El conocimiento específico de las características morfológicas del hígado y en especial sus aspectos celulares cuantitativos, resulta fundamental para evaluar el estado normal o patológico de éste órgano. La estereología permite realizar una reconstrucción tridimensional y una estimación cuantitativa de estructuras a nivel microscópico. Si bien es cierto, se han realizado numerosos estudios del hígado en varias especies, en los cuales se ha analizado la acción de diversos agentes, hay pocas investigaciones que permitan evaluar en modelos animales, los cambios morfológicos cuantitativos producidos en el hígado después de realizada una ovariectomía.

El objetivo de este estudio fue determinar aspectos cuantitativos del tejido hepático de ratas sometidas a un modelo experimental de menopausia inducida por ovariectomía.

\section{MATERIAL Y MÉTODO}

Utilizamos 10 ratas Sprague-Dawley (Rattus norvergicus) hembras adultas, sanas y mantenidas ad libitum en el Bioterio de la Universidad de Talca, Chile. Fueron pesadas y divididas en dos grupos de 5 ratas cada uno. Un grupo fue sometido a cirugía de ovariectomía bilateral (Grupo Ovx) y otro grupo a cirugía con la finalidad de exponer las ratas al mismo estrés quirúrgico que el grupo experimental (Grupo Sham). Luego de 60 días, las ratas fueron sacrificadas por dislocación cervical, previa anestesia con Ketamina ${ }^{\circledR}(5,0 \mathrm{mg} / \mathrm{kg})$. A través de laparotomía, fue retirado el hígado, determinando su volumen por el método de Scherle (1970). Obtuvimos aleatoriamente 1 trozo de cada hígado, siguiendo las reglas del Orientator (Mattfeldt et al.,1990). Las secciones fueron fijadas en formalina tamponada al $10 \%$, deshidratadas e incluidas en parafina para obtener 5 cortes histológicos de cada muestra con un grosor de $4 \mu \mathrm{m}(\mathrm{t}=0,004 \mathrm{~mm})$, las que fueron teñidos con HE. Se analizaron 5 campos por lámina, 125 campos en total. Las láminas fueron observadas en un microscopio óptico Olympus ${ }^{\circledR}$ modelo CX31, con cámara marca Moticam ${ }^{\circledR}$ modelo 480. Las imágenes fueron proyectadas en un monitor de pantalla plana marca Sony®. Para realizar la estereología se usó el test multipropósito M42.

Los parámetros estereológicos medidos fueron: densidad de número (Nv), densidad de volumen ( $\mathrm{Vv}$ ), densidad de superficie ( $\mathrm{Sv}$ ) y número total de hepatocitos y sinusoides hepáticos. Se calcularon, el promedio, la desviación estándar, el error estándar, los coeficientes de variación y de error. Para el análisis estadístico se utilizó el programa Stata versión 11,0 y se aplicó un análisis de varianza simple (ANOVA) para calcular el valor de p para todas las variables. Se consideró un índice de significancia menor o igual a 0,05. 


\section{RESULTADOS}

El peso promedio del grupo Ovx y Sham fue $300,8 \mathrm{~g}$ y $285,8 \mathrm{~g}$ respectivamente. La densidad de número promedio de hepatocitos en el hígado de rata sometida a cirugía Sham y Ovx fue de $6,89 \times 10^{5} / \mathrm{mm}^{3}$ (D.E. 1,14 ) y $7,70 \times 10^{5} /$ $\mathrm{mm}^{3}$ (D.E. 1,12) respectivamente. El valor de p para la $\mathrm{Nv}$ que compara ambos grupos fue 0,0001. La Nv de los hepatocitos para cada grupo se observa en la Fig. 1. El análisis estadístico de la Nv de los hepatocitos para las ratas sometidas a cirugía Sham y Ovx se muestra en la Tabla I.

La densidad de volumen promedio de hepatocitos en el hígado de rata sometida a cirugía Sham y cirugía Ovx fue de $71,92 \%$ (D.E. 4,37 ) y $8,48 \%$ (D.E. 4,53 ) respectivamente. El valor de p para la Vv que compara ambos grupos fue 0,0001 . El porcentaje de hepatocitos en las 5 muestras de ambos grupos se observa en la Fig. 2. El análisis estadístico para el Vv de los hepatocitos de hígados de ratas sometidas a cirugía Sham y cirugía Ovx se muestra en la Tabla II.
La densidad de superficie promedio ocupada por hepatocitos en el hígado de rata sometida a cirugía Sham y Ovx fue de $260,61 \mathrm{~mm}^{2} / \mathrm{mm}^{3}$ (D.E. 16,56) y $273,64 \mathrm{~mm}^{2} /$ $\mathrm{mm}^{3}$ (D.E. 19,01) respectivamente. El valor de p para la $\mathrm{Sv}$ que compara ambos grupos fue 0,0001. La densidad de superficie y el análisis estadístico para las 5 muestras de ambos grupos se observa en la Fig. 3 y Tabla III respectivamente.

El volumen promedio de los hígados de rata sometidos a cirugía Sham fue de $8,40 \mathrm{~mm}^{3}$ (D.E. 0,35) y el número total de hepatocitos fue en promedio 5,79 x $10^{6}$ (D.E. 0,31). El volumen promedio de los hígados de rata sometidos a cirugía Ovx fue $9,28 \mathrm{~mm}^{3}$ (D.E. 0,36) y el número total de hepatocitos fue en promedio $7,15 \times 10^{6}$ (D.E. 0,64).

Respecto a los sinusoides, la densidad de número promedio en el hígado de rata sometida a cirugía Sham y Ovx fue de $2,81 \times 10^{5} / \mathrm{mm}^{3}$ (D.E. 0,99 ) y $2,64 \times 10^{5} / \mathrm{mm}^{3}$ (D.E. $0,99)$ respectivamente. El valor de p para la Nv que compa-

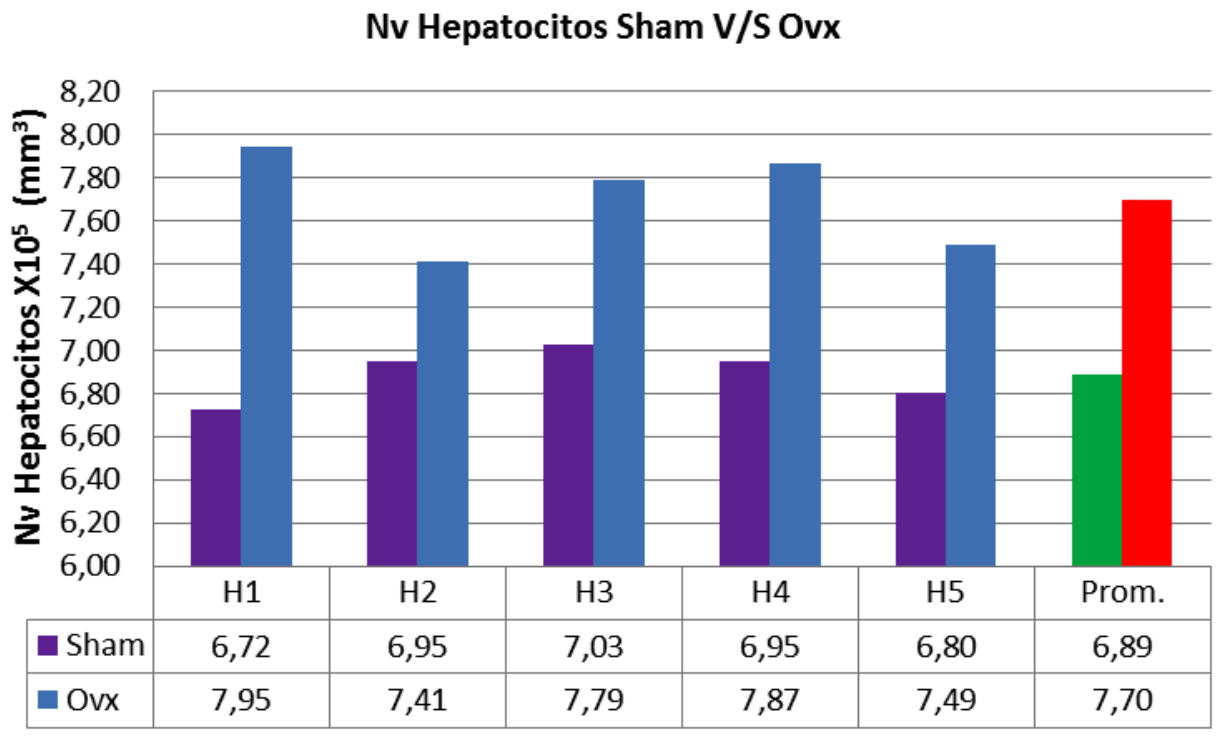

Fig. 1. Densidad de número de hepatocitos de hígado de rata (Rattus novergicus) sometida a cirugía Sham y cirugía Ovx.

Tabla I. Densidad de número promedio de hepatocitos en el hígado de rata (Rattus novergicus) sometida a cirugía Sham y cirugía Ovx.

\begin{tabular}{lcccccrrr}
\hline Nv & $\begin{array}{c}\text { Promedio } \\
\left(\text { células } / \mathrm{mm}^{3}\right)\end{array}$ & D.E. & $\begin{array}{c}\text { Mínimo } \\
\left(\text { células } / \mathrm{mm}^{3}\right)\end{array}$ & $\begin{array}{c}\text { Máximo } \\
\left(\text { células } / \mathrm{mm}^{3}\right)\end{array}$ & $\begin{array}{c}\text { E.E. } \\
\left(\text { células } / \mathrm{mm}^{3}\right)\end{array}$ & $\begin{array}{c}\text { C.V. } \\
(\%)\end{array}$ & $\begin{array}{c}\text { C.E. } \\
(\%)\end{array}$ & $\begin{array}{c}\text { Valor de } \\
\text { p }\end{array}$ \\
\hline Hep. Sham & $6,89 \times 105$ & 1,14 & $5,81 \times 105$ & $8,02 \times 105$ & $0,51 \times 10^{5}$ & 16,47 & 7,36 & 0,8868 \\
Hep. Ovx & $7,70 \times 105$ & 1,12 & $9,50 \times 105$ & $12,67 \times 105$ & $0,70 \times 10^{5}$ & 14,19 & 6,35 & 0,5107 \\
\hline
\end{tabular}


TRUJILLO, E.; VÁSQUEZ, B. \& DEL SOL, M. Características estereológicas del hígado de rata (Rattus norvegicus) sometidas a menopausia inducida por ovariectomía. Int. J. Morphol., 29(4):1470-1478, 2011.

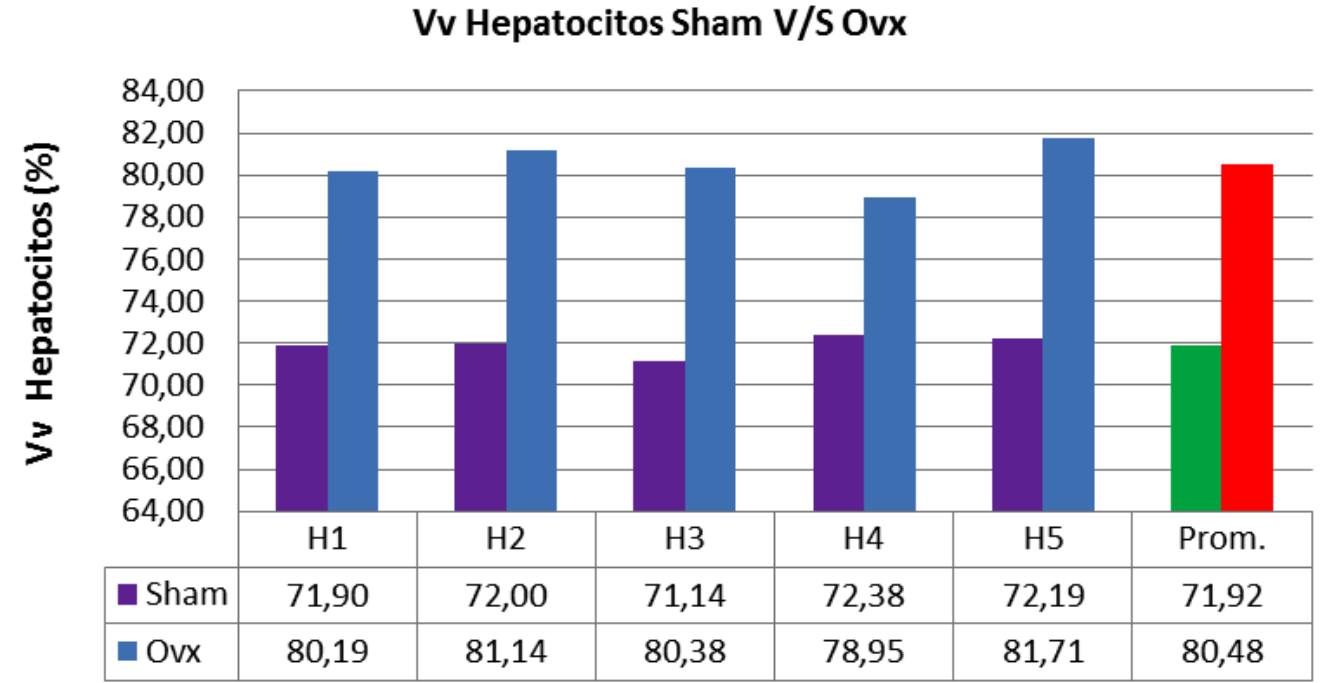

Fig. 2. Densidad de volumen de hepatocitos de hígado de rata (Rattus novergicus) sometida a cirugía Sham y cirugía Ovx.

Tabla II. Densidad de volumen promedio de hepatocitos en el hígado de rata (Rattus novergicus) sometida a cirugía Sham y cirugía Ovx.

\begin{tabular}{|c|c|c|c|c|c|c|c|c|c|}
\hline $\mathrm{Vv}$ & Promedio \% & D.E. & Mínimo \% & Máximo \% & E.E. & $\%$ & C.V. $(\%)$ & C.E. $\quad(\%)$ & Valor de $\mathrm{p}$ \\
\hline Hep. Sham & 71,92 & 4,37 & 66,88 & 76,88 & 1,95 & & 6,07 & 2,71 & 0,8950 \\
\hline Hep. Ovx & 80,48 & 4,53 & 75,16 & 85,62 & 2,03 & & 5,64 & 2,52 & 0,3109 \\
\hline
\end{tabular}

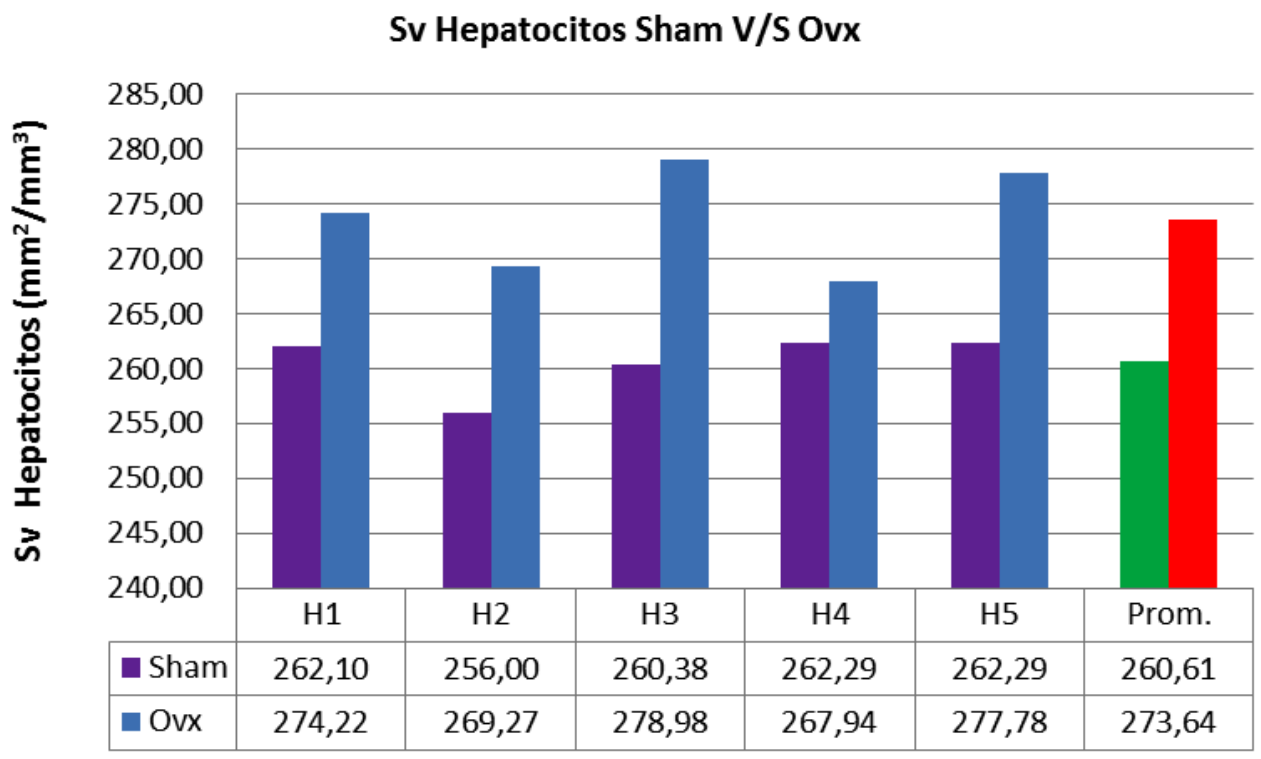

Fig. 3. Densidad de superficie de hepatocito de hígado de rata (Rattus novergicus) sometida a cirugía Sham y cirugía Ovx.

Tabla III. Densidad de superficie promedio de hepatocitos en el hígado de rata sometida a cirugía Sham y cirugía Ovx.

\begin{tabular}{|c|c|c|c|c|c|c|c|c|}
\hline $\mathrm{Sv}$ & $\begin{array}{l}\text { Promedio } \\
\left(\mathrm{mm}^{2} / \mathrm{mm}^{3}\right)\end{array}$ & D.E. & $\underset{\left(\mathrm{mm}^{2 /} \mathrm{mm}^{3}\right)}{\text { Mínimo }}$ & $\begin{array}{c}\text { Máximo } \\
\left(\mathrm{mm}^{2} / \mathrm{mm}^{3}\right)\end{array}$ & $\begin{array}{c}\text { E.E. } \\
\left(\mathrm{mm}^{2} / \mathrm{mm}^{3}\right)\end{array}$ & $\begin{array}{l}\text { C.V. } \\
(\%)\end{array}$ & $\begin{array}{l}\text { C.E. } \\
(\%)\end{array}$ & $\begin{array}{c}\text { Valor de } \\
\text { p }\end{array}$ \\
\hline Hep. Sham & 260,61 & 16,56 & 241,40 & 277,66 & 7,41 & 6,36 & 2,84 & 0,6450 \\
\hline Hep. Ovx & 273,64 & 19,01 & 251,33 & 295,70 & 8,50 & 6,94 & 3,11 & 0,1446 \\
\hline
\end{tabular}


ra ambos grupos fue 0,1610 . La Nv de los sinusoides en los 5 grupo se observa en la Fig. 4. El análisis estadístico de la
Nv de los sinusoides para las ratas sometidas a cirugía Sham y cirugía Ovx se muestra en la Tabla IV.

\section{Nv Sinusoides Sham V/S Ovx}

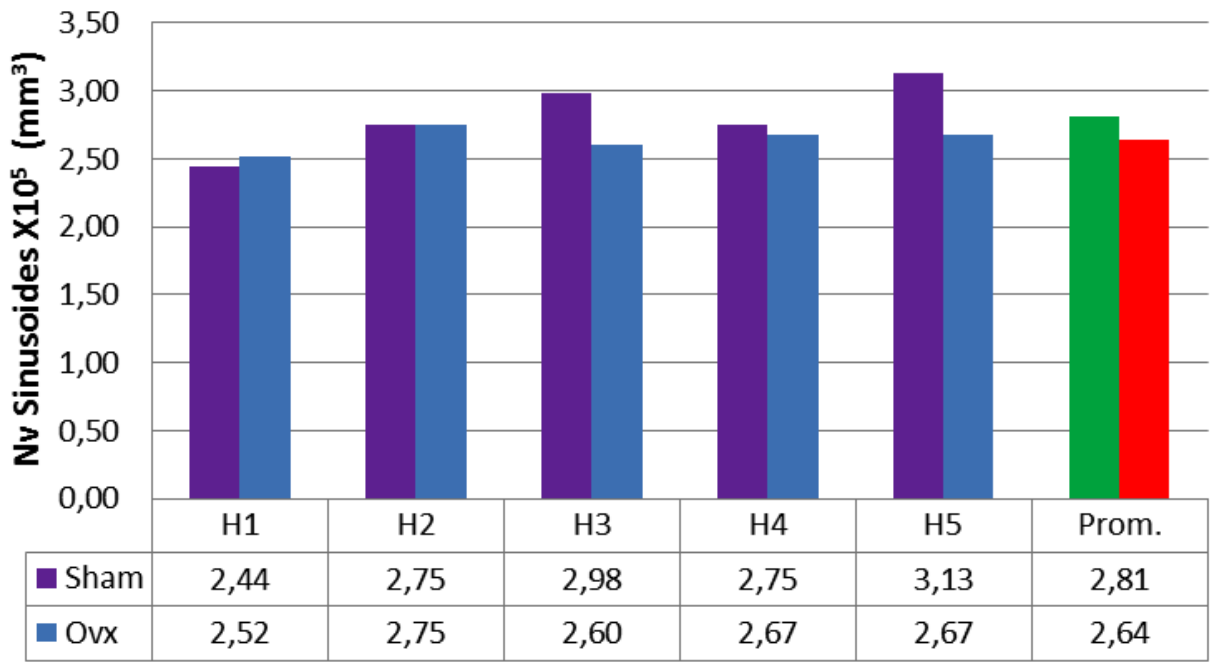

Fig. 4. Densidad de número de sinusoides de hígado de rata (Rattus novergicus) sometida a cirugía Sham y cirugía Ovx.

Tabla IV. Densidad de número promedio de sinusoides en el hígado de rata (Rattus novergicus) sometida a cirugía Sham y cirugía Ovx.

\begin{tabular}{|c|c|c|c|c|c|c|c|c|}
\hline $\mathrm{Nv}$ & $\begin{array}{c}\text { Promedio } \\
\left(\text { sinusoide } / \mathrm{mm}^{3}\right)\end{array}$ & D.E. & $\begin{array}{c}\text { Mínimo } \\
\left(\text { sinusoide } / \mathrm{mm}^{3}\right)\end{array}$ & $\begin{array}{c}\text { Máximo } \\
\left(\text { sinusoide } / \mathrm{mm}^{3}\right)\end{array}$ & $\begin{array}{c}\text { E.E. } \\
\left(\text { sinusoide } / \mathrm{mm}^{3}\right)\end{array}$ & $\begin{array}{l}\text { C.V. } \\
(\%)\end{array}$ & $\begin{array}{l}\text { C.E. } \\
(\%)\end{array}$ & $\begin{array}{l}\text { Valor } \\
\text { de p }\end{array}$ \\
\hline Sinusoide Sham & $2,81 \times 10^{5}$ & 0,99 & $1,96 \times 10^{5}$ & $3,79 \times 105$ & $0,44 \times 10^{5}$ & 35,47 & 15,86 & 0,1118 \\
\hline Sinusoide Ovx & $2,64 \times 10^{5}$ & 0,99 & $1,96 \times 10^{5}$ & $3,77 \times 105$ & $0,44 \times 10^{5}$ & 37,56 & 16,80 & 0,9310 \\
\hline
\end{tabular}

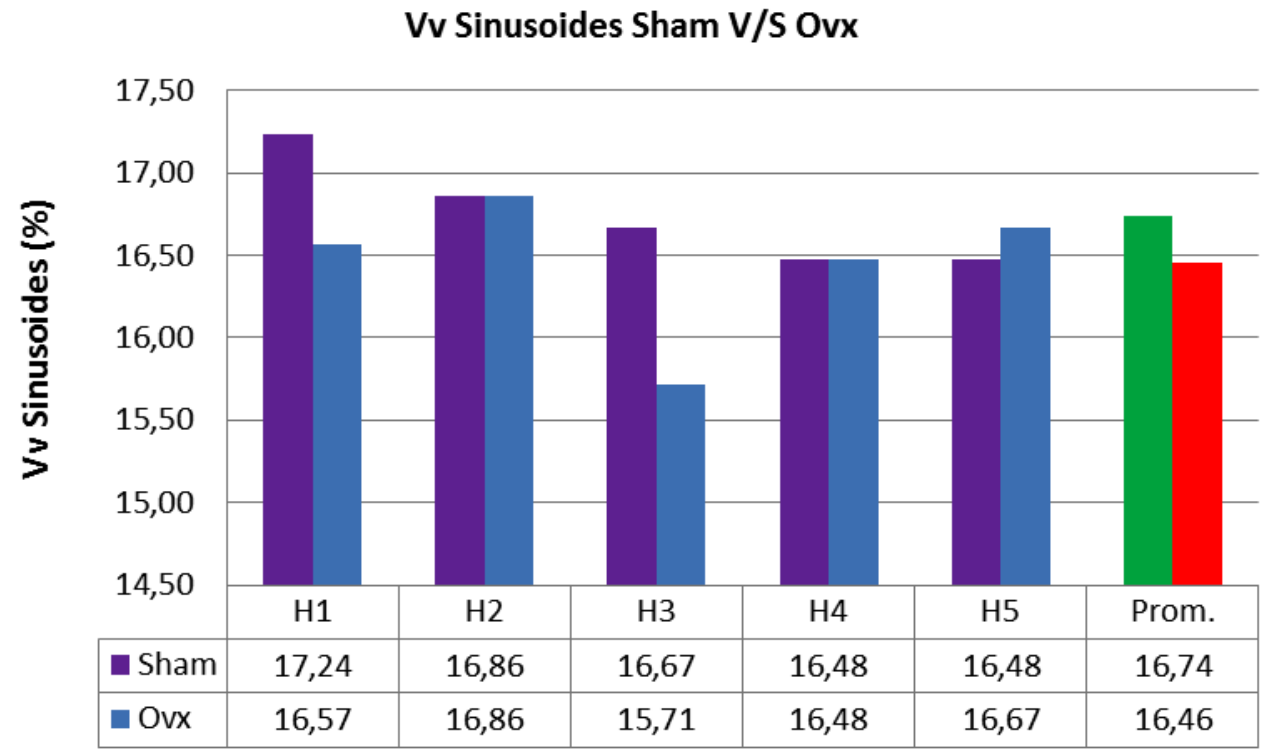

Fig. 5. Densidad de volumen de sinusoides en hígado de rata (Rattus novergicus) sometida a cirugía Sham y cirugía Ovx.

Tabla V. Densidad de volumen promedio de sinusoides en el hígado de rata sometida a cirugía Sham y cirugía Ovx.

\begin{tabular}{lccccccccc}
\hline Vv & Promedio $\%$ & D.E. & Mínimo $\%$ & Máximo $\%$ & E.E. \% & C.V. $(\%)$ & C.E. (\%) & Valor de p \\
\hline Sinusoide Sham & 16,74 & 2,10 & 14,57 & 19,29 & 0,94 & 12,52 & 5,60 & 0,7237 \\
Sinusoide Ovx & 16,46 & 2,04 & 14,13 & 18,76 & 0,91 & 12,41 & 5,55 & 0,3719 \\
\hline
\end{tabular}




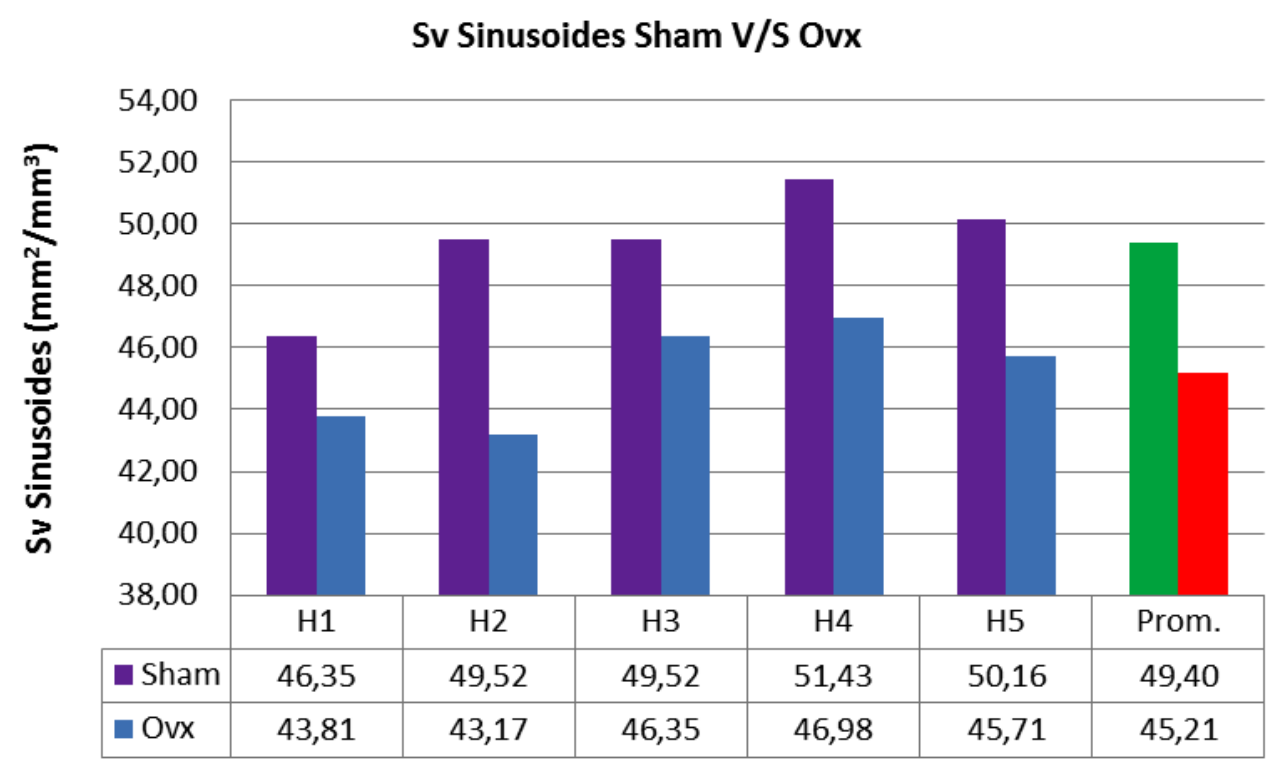

Fig. 6. Densidad de superficie de sinusoides en hígado de rata (Rattus novergicus) sometida a cirugía Sham y cirugía Ovx.

Tabla VI. Densidad de superficie promedio de sinusoides en el hígado de rata (Rattus novergicus) sometida a cirugía Sham y cirugía Ovx.

\begin{tabular}{lcccccccc}
\hline Sv & $\begin{array}{c}\text { Promedio } \\
\left(\mathrm{mm}^{2} / \mathrm{mm}^{3}\right)\end{array}$ & D.E. & $\begin{array}{c}\text { Mínimo } \\
\left(\mathrm{mm}^{\left.2 / \mathrm{mm}^{3}\right)}\right.\end{array}$ & $\begin{array}{c}\text { Máximo } \\
\left(\mathrm{mm}^{2} / \mathrm{mm}^{3}\right)\end{array}$ & $\begin{array}{c}\text { E.E. } \\
\left(\mathrm{mm}^{2} / \mathrm{mm}^{3}\right)\end{array}$ & $\begin{array}{c}\text { C.V. } \\
(\%)\end{array}$ & $\begin{array}{c}\text { C.E. } \\
(\%)\end{array}$ & $\begin{array}{c}\text { Valor de } \\
\mathrm{p}\end{array}$ \\
\hline Sinusoide Sham & 49,40 & 11,31 & 36,83 & 61,84 & 5,06 & 22,92 & 10,25 & 0,6335 \\
Sinusoide Ovx & 45,21 & 8,63 & 37,46 & 55,24 & 3,86 & 19,12 & 8,55 & 0,5839 \\
\hline
\end{tabular}

La densidad de volumen promedio de sinusoides en el hígado de rata sometida a cirugía Sham y Ovx fue de $16,74 \%$ (D.E. 2,10 ) y $16,46 \%$ (D.E. 2,04 ) respectivamente. El valor de p para la Vv que compara ambos grupos fue 0,2965 . El porcentaje de sinusoides hepáticos en las 5 muestras de ambos grupos se observa en la Fig. 5. El análisis estadístico para el Vv de los sinusoides de hígado de rata sometidas a cirugía Sham y cirugía Ovx se muestra en la Tabla V.

La densidad de superficie promedio ocupada por los sinusoides en el hígado de rata sometida a cirugía Sham y Ovx fue de $49,40 \mathrm{~mm}^{2} / \mathrm{mm}^{3}$ (D.E. 11,31) y $45,21 \mathrm{~mm}^{2} / \mathrm{mm}^{3}$ (D.E. 8,63), respectivamente. El valor de p para la Sv que compara ambos grupos fue 0,0022 . La densidad de superficie y el análisis estadístico para las 5 muestras de ambos grupos se observa en la Fig. 6 y Tabla VI, respectivamente.

El número total de sinusoides hepáticos de ratas sometidas a cirugía Sham y cirugía Ovx fue en promedio 2,37 x 106 (D.E. 0,30) y 2,45 x 106 (D.E. 0,11), respectivamente.

\section{DISCUSIÓN}

Es sabido que la ovariectomía en ratas adultas produce un aumento en la ingesta y disminución de la actividad física seguida de un incremento en el peso corporal y adiposidad (Wade \& Gray, 1979; Richard, 1986; Shimizu, 2003; Mayes \& Watson; Lavoie et al.,). Una prueba indirecta respecto a que el estradiol incrementa la oxidación de lípidos es la aparición de hígado graso y esteatosis hepática en mujeres tratadas con tamoxifeno, un potente antiestrógeno (Saibara et al., 1999). Por su parte, Nemoto et al., demostraron la presencia de esteatosis hepática en ratas y ratones deficientes en la enzima aromatasa o la desaparición tras el tratamiento con estradiol (Hewitt et al., 2004).

El estudio histopatológico del hígado permite realizar una adecuada evaluación diagnóstica y conocer de manera más directa y específica las causas y consecuencias de estas alteraciones (Angulo). Además, este método diagnóstico ofrece otras ventajas, ya que permite profundizar el aná- 
lisis de las muestras a través de evaluaciones cuantitativas estereológicas más precisas respecto a los cambios morfológicos sufridos por el hígado como consecuencia de la disminución de la acción hormonal estrogénica. De esta manera, podemos conocer parámetros tales como densidad de número, de volumen y de superficie, de los distintos elementos que conforman este órgano.

Por tal razón, existen numerosos estudios que evalúan parámetros estereológicos hepáticos en diversas especies, incluidas las ratas. Sin embargo, son pocos los que analizan cambios morfológicos aplicados al modelo experimental por ovariectomía. Es importante señalar además, que los parámetros esterelógicos reportados por diversos autores son muy variados, este hecho puede deberse a factores tales como edad, raza, alimentación, enfermedades, entre otros. Así, Weibel et al.; Carthew et al. (1998) y Martins et al. (2005) determinaron una cantidad superior de hepatocitos por $\mathrm{mm}^{3}$ con respecto a lo observado en nuestro estudio. Sin embargo, Águila et al., reportaron valores menores en ratas sometidas a altas concentraciones de lípidos en la dieta. Noorafshan et al., (2005); Moreti et al. (2005); Silva et al., (2006); Ragonha et al.; Marcos et al., (2006) y Valenca et al., observaron valores inferiores a los 7,7 x $10^{5}$ células $/ \mathrm{mm}^{3}$ encontrados en ratas sometidas a cirugía Ovx y a los 6,89x10 células $/ \mathrm{mm}^{3}$ encontrados en ratas sometidas a cirugía Sham. Otras especies, como el cobayo (Cavia porcellus) presentan valores de $\mathrm{Nv}$ de 9,20x10 células $/ \mathrm{mm}^{3}$ (Rosas et al., 2007), resultados levemente superiores tanto al grupo Sham como al grupo Ovx.

Respecto al porcentaje de hepatocitos presentes en el hígado de rata, Weibel et al., reportaron un $83,1 \%$ en ratas Wistar, resultado superior al 71,92\% obtenido en el grupo Sham, pero bastante similar al 80,48\% del grupo Ovx. Ragonha et al., en hígado de ratones Swiss Albino encontraron un Vv de 76,17\% de hepatocitos, resultado levemente superior al grupo Sham, pero inferior al grupo Ovx y Neves et al., (2006) reportaron un Vv sobre el $90 \%$ de hepatocitos en hígado de ratones, muy superior a lo observado en ambos grupos de nuestro trabajo. Blouin et al., informaron un $77,8 \%$, de hepatocitos en el parénquima hepático de ratas Sprague-Dawley, considerando que el parénquima representa un $92,4 \%$ del volumen del hígado. Por lo tanto, el Vv de hepatocitos fue de un $71,89 \%$, resultados muy similares al grupo Sham e inferiores al grupo Ovx. Por su parte, Águila et $a l$, demostraron que una alta concentración de lípidos en la dieta, no solo causa esteatosis hepática, sino que también, afecta las proporciones entre hepatocitos y otros tejidos en el hígado, por lo que sus resultados fueron bastante inferiores a los nuestros. Moreti et al. y Valenca et al., señalaron una densidad de volumen de $30,72 \%$ y $48 \%$ respectivamente, muy por debajo de nuestros resultados. Barros et al. (2009) reportaron un $\mathrm{Vv}$ de $71,7 \%$ de hepatocitos en hígados de ratas Swiss Webster, resultados muy similares a los nuestros.

Respecto al valor de p obtenido en nuestros resultados, es importante destacar que para todos los parámetros estereológicos analizados, tanto para el grupo Sham como para el grupo Ovx, fue mayor a 0,05, lo cual indica que no existió diferencia significativa entre los resultados de los individuos que constituían cada grupo. Sin embargo, al comparar los valores de p para el número de hepatocitos por $\mathrm{mm} 3$, porcentaje de hepatocitos y superficie que ocupan éstos en el hígado entre el grupo Sham y Ovx, existió siempre un valor de $\mathrm{p}<0,05$, lo que indica diferencias significativas entre los resultados. Así, la Nv de los hepatocitos del grupo Ovx $\left[7,70 \times 10^{5} / \mathrm{mm}^{3} \quad\right.$ (D.E. 1,12) $]$ aumentó significativamente en relación a lo observado en el grupo Sham $\left[6,89 \times 10^{5} / \mathrm{mm}^{3}\right.$ (D.E. 1,14)]. Estos resultados podrían explicarse con lo reportado por otros autores. Shimizu (2003) y Codes et al., (2007b) señalaron que la disminución de la función ovárica se asocia con aumentos en los niveles séricos de citoquinas proinflamatorias como las interleucina 1 (IL1) y la interleucina 6 (IL-6). Por su parte, Camargo et al., demostraron que estas citoquinas podrían desempeñar un papel importante en la promoción de la regeneración hepática. Por otro lado, al comparar los parámetros estereológicos de los sinusoides hepáticos entre ambos grupos, se observó que en promedio la Nv, Vv y Sv fue menor en el grupo Ovx. Sin embargo, esta diferencia fue significativa solo para la Sv de los sinusoides hepáticos.

Estos resultados nos sugieren que la disminución o ausencia de estrógeno no solo puede generar hígado graso y esteatósis hepática (Hewitt et al. y Saibara et al.), sino que también puede generar cambios importantes en la organización y proporción de los distintos componentes del hígado que podrían ser factores importantes a considerar al momento del diagnóstico y pronóstico de alteraciones hepáticas.

TRUJILLO, E.; VÁSQUEZ, B. \& DEL SOL, M. Stereological characteristics of the liver of rats (Rattus norvegicus) submitted to ovariectomy-induced menopause. Int. J. Morphol., 29(4):14701478, 2011.

SUMMARY: During menopause, hormonal changes such as decreased estradiol and increased follicle stimulating and luteinizing hormones occur. In premenopausal women fibrosis and hepatic steatosis is less than in postmenopausal women and improves when hormone replacement therapy is administered. The aim of this study was to determine quantitative aspects of the liver of rats undergoing an experimental model of ovariectomy-induced menopause. We used 10 Sprague-Dawley (Rattus norvergicus) healthy adults. The rats were weighed and divided into two groups 
(bilateral oophorectomy = Group Ovx and control = Group Sham). The animals were sacrificed at 60 days and the liver removed, obtaining a piece of each liver. Five cuts were made of 4 microns and stained with HE. We analyzed five fields per slide. M42 test was used. Stereological parameters considered were as follows: Nv, $\mathrm{Vv}, \mathrm{Sv}$ and total number (Tn) of hepatocytes and sinusoids. The average weight of Ovx and Sham group was $300.8 \mathrm{~g}$ and $285.8 \mathrm{~g}$, respectively. The $\mathrm{Nv}$ of hepatocytes of rats subjected to sham surgery (CS) and Ovx (CO) was 6.89 and $7.70 \times 10^{5} / \mathrm{mm}^{3} \times 10^{5} / \mathrm{mm}^{3}$, respectively. The Vv of CS and CO hepatocytes was $71.92 \%$ and $80.48 \%$, respectively. SV and CO, CS hepatocytes was $260.61 \mathrm{~mm}^{2} /$ $\mathrm{mm}^{3}$ and $273.64 \mathrm{~mm}^{2} / \mathrm{mm}^{3}$, respectively. The average volume of CS livers was $8.40 \mathrm{~mm}^{3}$ and hepatocyte Tn averaged $5.79 \times 10^{6}$. The average volume was $9.28 \mathrm{~mm}^{3} \mathrm{CO}$ livers and Tn of $7.15 \times 10^{6}$. Regarding the sinusoids, the $\mathrm{Nv}$ in the liver CS and $\mathrm{CO}$ was 2.81 $\mathrm{x} 10^{5} / \mathrm{mm}^{3}$ and $2.64 \times 10^{5} / \mathrm{mm}^{3}$, respectively. The Vv of sinusoids in CS and CO was $16.74 \%$ and $16.46 \%$, respectively. The sinusoids in rats Sv CS and CO was $49.40 \mathrm{~mm}^{2} / \mathrm{mm}^{3}$ and $45.21 \mathrm{~mm}^{2} / \mathrm{mm}^{3}$, respectively. The sinusoids Tn CS and CO averaged $2.37 \times 10^{6}$ and $2.45 \times 10^{6}$, respectively. The lack of estrogen can cause fibrosis and hepatic steatosis as noted by other authors, but also changes in the organization and the proportion of the components of the liver, factors to consider during diagnosis and prognosis of liver disorders.

KEYWORDS: Ovariectomy-induced Menopause; Liver; Stereology; Rattus norvergicus.

\section{REFERENCIAS BIBLIOGRÁFICAS}

Águila, M. B.; Pinheiro, A. R.; Parente, L. B. \& Mandarim-deLacerda, C. A. Dietary effect of different high-fat diet on rat liver stereology. Liver International, 23: 363-70, 2003.

Angulo, P. Non alcoholic fatty liver disease. N. Engl. J. Med. 346:1221-1231, 2002.

Barros, L. A.; Costa-Silva, M.; Biolchini, C. L.; Neves, R. H. \& Machado-Silva J.R.. Effect of praziquantel administration on hepatic stereology of mice infected with Schistosoma mansoni and fed a low-protein diet. Braz. J. Med. Bio.l Res., 42(9):8125, 2009.

Blouin, A.; Bolender, R. P. \& Weibel, E. R. Distribution of organelles and membranes between hepatocytes and nonhepatocytes in rat liver parenchyma - a stereological study. J. Cell. Biol., 72:441-55, 1977.

Camargo, C.A.; Madden, J. F.; Gao, W.; Selvan, R. S. \& Clavien, P. Interleukin-6 protects liver against warm ischemia/ reperfusion injury and promotes hepatocyte proliferation in the rodent. Hepatology, 26(6):1513-20, 1997.

Carthew, P.; Edwards, R. E. \& Nolan, B. M. The quantitative distinction of hyperplasia from hypertrophy in hepatomegaly induced in the rat liver by phenobarbital. Toxicol. Sci., 44:4651,1998 .
Codes, L.; Asselah, T.; Cazals-Hatem, D.; Tubach, F.; Vidaud, D.; Paraná, R.; Bedossa, P.; Valla, D. \& Marcellin, P. Liver fibrosis in women with chronic hepatitis $\mathrm{C}$ : evidence for the negative role of the menopause and steatosis and the potential benefit of hormone replacement therapy. Gut. 56:390-5, 2007 a.

Codes, L.; Matos, L. \& Parana, R. Chronic hepatitis C and fibrosis: evidences for possible estrogen benefits. Braz. J. Infect. Dis., 11:371-4, 2007 b.

Di Martino, V.; Lebray, P.; Myers R. P.; Pannier, E.; Paradis, V.; Moussalli, J.; Thabut, D.; Buffet, C. \& Poynard, T. Progression of liver fibrosis in women infected with hepatits $\mathrm{C}$ : long-term benefit of estrogen exposure. Hepatology. 40:1426-33, 2004.

Hewitt, K. N.; Pratis, K.; Jones, M. E. \& Simpson, E. R. Estrogen replacement reverses the hepatic steatosis phenotype in the male aromatase knockout mouse. Endocrinology, 145:18428, 2004.

Lavoie, J. M. \& Pighon, A. NAFLD, Estrogens, and Physical Exercise: The Animal Model. J. Nutr. Metab., 2012:914-38, 2011.

Lip G. Y.; Blann, A. D.; Jones, A. F. \& Beevers, D. G. Effects of hormone-replacement therapy on hemostatic factors, lipid factors, and endothelial function in women undergoing surgical menopause: implications for prevention of atherosclerosis. Am. Heart J., 134:764-71, 1997.

Malarkey, D. E.; Johnson, K.; Ryan, L.; Boorman, G. \& Maronpot, R.R. New insights into functional aspects of liver morphology. Toxicol. Pathol., 33(1):27-34, 2005.

Marcos, R.; Monteiro, R. A. F. \& Rocha, E. Design-based stereological estimation of hepatocyte number, by combing the smooth optical fractionator and immunocytochemistry with anti-carcinoembryonic antigen polyclonal antibodies. Liver International, 26:116-124, 2006.

Martins, A. T.; Azoubel, R.; Lopes, R. A.; Di Matteo, M. A. S. \& Arruda, J. G. F. Effect of sodium cyclamate on the rat fetal liver: A karyometric and stereological study. Int. J. Morphol., 23(3):221-6, 2005.

Mattfeldt, T.; Mall, G.; Gharehbaghi, H.; Moller, P. Estimation of surface area and length with the orientator. J. Microsc., 159 (3):301-17, 1990.

Mayes, J. S. \& Watson, G. H. Direct effects of sex steroid hormones on adipose tissues and obesity. Obesity Reviews., 5(4):197216, 2004.

Moreti, D. L. C.; Lopes, R. A.; Vinha, D.; Sala, M. A.; Semprini, M. \& Friedrichi, C. Efectos del albendazol en el hígado de feto de rata. Estudios morfológico y morfométrico. Int. J. Morphol., 23(2):111-20, 2005 
Nemoto. Y.; Toda, K.; Ono, M. Fujikawa-Adachi, K.; Saibara, T.; Onishi, S.; Enzan, H.; Okada, T. \& Shizuta, Y. Altered expression of fatty acid-metabolizing enzymes in aromatasedeficient mice. J. Clin. Invest., 105:1819-25, 2000.

Neves, R. H.; Alencar, A. C.; Aguila, M. B.; Mandarim-de-Lacerda, C. A.; Machado-Silva, J. R. \& Gomes, D. C. Hepatic stereology of schistosomiasis mansoni infected-mice fed a high-fat diet. Mem. Inst. Oswaldo Cruz, 101(1):253-60, 2006.

Noorafshan A, Esmail-Zadeh B, Bahmanpour S, Poost-Pasand A. Early stereological changes in liver of Sprague-Dawley rats after streptozotocin injection. Indian J. Gastroenterol. 24(3):104-7, 2005.

Ragonha, L. H. O.; Abrahao, A. A. C.; Sala, M. A.; Lopes, R. A.; Ribeiro, R. D.; Prado JR., J. C.; Albuquerque, S. \& Zucoloto, S. Estudio morfométrico del hígado de ratón en la enfermedad de Chagas experimental. Int. J. Morphol., 24(3):383-90, 2006.

Richard D. Effects of ovarian hormones on energy balance and brown adipose tissue thermogenesis. Am J Physiol., 250:2459, 1986.

Rosas C.; Vásquez B.; \& del Sol, M. Stereological Study of the Guinea-pig Liver (Cavia porcellus). Int. J. Morphol., 25(3):557-60, 2007.

Saibara, T.; Onishi, S.; Ogawa, Y.; Yoshida, S. \& Enzan, H. Nonalcoholic steatohepatitis. Lancet, 354:1299-300, 1999.

Santoro, N. The menopause transition: an update. Hum. Reprod. Update., 8:155-160, 2002.

Scherle, W. A simple method for volumetry of organs in quantitative stereology. Mikroskopie, 26:57-63, 1970.

Shimizu, I. Impact of estrogens on the progression of liver disease. Liver International, 23:63-9, 2003.

Shimizu, I. \& Ito, S. Protection of estrogens against the progression of chronic liver disease. Hepatology Research, 37:239-47, 2007.

Silva, R. F.; Lopes, R. A.; Sala, M. A.; Vinha, D.; Regalo, S. C. H.; Souza, A. M. \& Gregorio, Z. M. O. Action of trivalent chromium on rat liver structure. Histometric and haematological studies. Int. J. Morphol., 24(2):197-203, 2006.

Valenca, s. S.; Gouveia, L.; Pimenta, W. A. \& porto, 1. C. Effects of oral nicotine on rat liver stereology. Int. J. Morphol., 26(3):1013-22, 2008.

Wade, G. N. \& Gray, J. M. Gonadal effects on food intake and adiposity: a metabolic hypothesis. Physiol. Behav., 22:583-93, 1979.
Weibel, E. R.; Staubli, W.; Gnagi, H. \& Hess, F. A. correlated morphometric and biochemical studies on liver cell - I. Morphometric Model, Stereologic methods, and normal morphometric data for rat liver. J. Cell. Biol., 42:68-91, 1969.

Yasuda, M.; Shimizu, I.; Shiba, M \& Ito, S. Suppressive effects of estradiol on dimethylnitrosamine-induced fibrosis of the liver in rats. Hepatology, 29:719-27, 1999.

Zhou, Y.; Yin, D.; Chen, H. Shi, J.; Sha, B. \& Wang, X. Inhibitory effects of idoxifene on hepatic fibrosis in rats. Acta Pharmacologica Sinica, 26:581-6, 2005.

Dirección para correspondencia:
Erick Gonzalo Trujillo
Universidad de Talca
Lircay s/n
Talca
CHILE

Email: etrujillo@utalca.cl

Recibido: 10-09-2011

Aceptado 12-10-2011 